\title{
EL APRENDIZAJE COOPERATIVO COMO PROCEDIMENTO PARA DESARROLLAR LA COMPETENCIA COMUNICATIVA EN INGLÉS EN EL SISTEMA EDUCATIVO CUBANO
}

\section{COOPERATIVE LEARNING AS A PROCEDURE FOR DEVELOPING THE ENGLISH COMMUNICATIVE COMPETENCE IN THE CUBAN EDUCATIONAL SYSTEM}

\author{
Eduardo Escalona Pardo \\ Yusimí Frías Reyes \\ María Eugenia Fonseca Calderón \\ Universidad de Granma, Cuba
}

\begin{abstract}
Resumen
Este artículo tiene como objetivo presentar al aprendizaje cooperativo para desarrollar la competencia comunicativa en las clases de inglés como elemento indispensable en la formación de las futuras generaciones que exige el sistema educativo cubano en la actualidad. Para facilitar su comprensión, se parte del enfoque comunicativo como método fundamental en la enseñanza aprendizaje del idioma inglés, se ofrecen los conceptos de competencia y competencia comunicativa, y se sugieren novedosas técnicas y métodos que se pueden emplear en los grupos de aprendizaje cooperativos que se creen en las aulas. Se corrobora que los estudiantes presentan limitaciones a la hora de interactuar oralmente entre ellos y con los profesores, y que el empleo de estas novedosas técnicas y métodos contribuye a la solución de esta problemática.
\end{abstract}

Palabras clave: Competencia comunicativa, enfoque comunicativo, aprendizaje cooperativo, inglés, técnicas.

\begin{abstract}
The article is aimed at presenting cooperative learning to develop communicative competence in the English lessons as an important element in the formation of the future generations the Cuban educational system demands nowadays. To make its comprehension easier, it starts from the communicative approach as the fundamental method in teaching and learning English. The concepts of competence and communicative competence are stated, and some methods and cooperative learning techniques that can be used in the cooperative learning groups created in class are suggested. The authors corroborate the limitations students face when interacting orally with each other and with their professors, and that the use of these novel techniques and methods contributes to give a solution to this problem.
\end{abstract}

Key Words: Communicative competence, communicative approach, cooperative learning, English, techniques.

\section{INTRODUCCIÓN}

En la actual Política Educacional Cubana, dentro de las fundamentales áreas culturales de la educación para el desarrollo armónico de la personalidad de los estudiantes se encuentra la educación del lenguaje 
para la comunicación, incluyéndose tanto la lengua materna como una o más lenguas extranjeras. Debido a los cambios económicos ocurridos en el mundo, el desarrollo de la revolución científico-técnica, el comercio e intercambio cultural generado a raíz de la globalización, el gobierno cubano ha trazado una nueva política relacionada con el aprendizaje del idioma inglés. Se trata de dominar esta lengua extranjera una vez terminados los estudios universitarios. Los estudiantes deben enfrentarse a un examen de colocación y demostrar un adecuado nivel de competencia comunicativa.

Esta situación implica la aplicación de nuevas formas de enseñanza aprendizaje que garanticen el desarrollo de esta competencia en los estudiantes en las clases de idioma inglés. Al ser su enseñanza eminentemente comunicativa, se recurre al aprendizaje cooperativo como forma de garantizar la motivación y desarrollo de habilidades de trabajo en grupo que posteriormente se convierten en habilidades individuales positivas y competencias de diversos tipos.

Al entrevistar diferentes estudiantes de segundo año de la carrera Lenguas Extranjeras, Inglés, se conoció que les resulta difícil encontrar la gramática correcta y el vocabulario apropiado para usarlo de manera espontánea en una situación determinada de su realidad objetiva; se preocupan por los errores que pudieran cometer al expresarse oralmente; y muchas veces comprenden lo que los demás dicen, pero no saben interactuar con ellos. Se efectúa la evaluación del proceso de enseñanza aprendizaje en este nivel y los datos recabados permiten fundamentar y sentar las bases teóricas para el empleo de diferentes técnicas de aprendizaje cooperativo que permiten el desarrollo de la competencia comunicativa en las clases de inglés como lengua extranjera.

\subsection{Población y muestra}

Para el desarrollo de la investigación se tuvo en cuenta una población de 60 estudiantes de segundo año de la carrera Lenguas Extranjeras, Inglés en la Universidad de Granma, Cuba; distribuidos en tres subgrupos y 4 profesores de inglés. Como muestra se tomó de manera intencional el subgrupo 01 con una matrícula de 18 estudiantes, los cuales constituyen el $30 \%$ de la población. También fueron incluidos los cuatro profesores.

\subsection{Métodos}

Se ejecutó un estudio teórico desde el método analítico-sintético y la revisión bibliográfica para determinar los referentes teóricos relacionados con el aprendizaje cooperativo y el enfoque comunicativo aplicado en la enseñanza aprendizaje del idioma inglés en el sistema educativo cubano; así como como la observación, la entrevista, la encuesta, y el pre-experimento para corroborar la efectividad de las técnicas de aprendizaje cooperativo sugeridas para el desarrollo de la competencia comunicativa en las clases.

\section{ANÁLISIS Y DISCUSIÓN}

\subsection{El enfoque comunicativo. Fundamentos teóricos}

Según Edward (1963) y citada por Brown (1994), enfoque es un sistema de concepciones relacionadas con la naturaleza de la lengua, de su aprendizaje, y su enseñanza. Entonces se podrá comprender por 
qué se habla de "enfoque" comunicativo. Según Font (2005) se trata de una concepción educativa que se refleja en todo lo que sucede en el aula y que, como plantea Nunan (1989), está marcada por concepciones acerca de la naturaleza de la lengua y de su aprendizaje.

El objetivo de la enseñanza de la lengua es desarrollar lo que Hymes (1972) llamó "competencia comunicativa". Este término se utilizó con el propósito de contrastar una visión comunicativa del idioma con la teoría de competencia de Chomsky (1965).

De acuerdo con Richards (2001), para Chomsky el objetivo de la teoría lingüística era caracterizar las habilidades abstractas que posee el hablante para poder producir oraciones gramaticalmente correctas en una lengua. Hymes consideraba que esta concepción de teoría lingüística era estéril, y que la teoría lingüística necesitaba convertirse en parte de una teoría más general que incorporara la comunicación y la cultura.

El enfoque comunicativo surge en la década de 1970 y fue aceptado paulatinamente en los años 80 . Brown (1994) enmarca al enfoque comunicativo dentro de la teoría lingüística que considera al lenguaje como un sistema para la expresión de significado, de propósitos funcionales en un medio interactivo de comunicación. Utiliza actividades que involucran una comunicación real, desarrollando tareas significativas y utilizando un lenguaje que es, a su vez, significativo para el que aprende. Se aborda la lengua en su realización social concreta, aspecto fundamental para utilizar el aprendizaje colaborativo en las clases.

Dentro de este enfoque, los objetivos de aprendizaje reflejan las necesidades del estudiante, e incluirán tanto habilidades funcionales (uso social de la lengua) como competencia lingüística. El aprendizaje de una lengua presupone su empleo social, por eso es inseparable de la comprensión de las otras personas, el entorno y de la experiencia social e individual (Vygotsky, 1984).

El enfoque comunicativo reconoce como su objetivo el logro de la competencia comunicativa, definida por Savignon (1983) como la eficiencia funcional en el uso del lenguaje; la expresión, interpretación y negociación de significado que involucra la interacción de dos o más personas que pertenecen a la misma o a diferentes comunidades lingüísticas, o entre una persona y un texto oral o escrito.

Richards (2001) define la competencia comunicativa como la capacidad de usar el lenguaje apropiadamente en la comunicación en dependencia del contexto espacial y temporal, de los roles de los participantes, y de la naturaleza de la transacción que se produce.

Los autores de este artículo asumen el concepto de competencia comunicativa ofrecido por Zimniaya (1989) quien la considera como el conjunto de reglas sociales, de la cultura nacional, las valoraciones y valores que determinan tanto la forma, como el contenido admisible en el lenguaje de la lengua que se estudia. Constituye la capacidad real individual de organizar la actividad verbal de forma adecuada a las diferentes situaciones de la comunicación (según los objetivos, las relaciones de roles, la forma y el contenido) en sus actividades receptivas y productivas, en correspondencia con cada situación 
concreta. Esta definición sustenta la aplicación del aprendizaje colaborativo para el logro de la competencia comunicativa en los estudiantes, pues al crear diferentes grupos de aprendizaje cooperativo, se asignan roles que permiten interactuar en situaciones comunicativas concretas.

Font (2005) plantea que para un mejor estudio y comprensión del concepto de competencia comunicativa se tienen en cuenta los siguientes componentes:

La competencia lingüística: también conocida tradicionalmente como competencia gramatical, se refiere a la habilidad del que usa la lengua extranjera para utilizar y comprender con corrección los tres niveles de la misma: la morfo-sintaxis, la pronunciación y el léxico.

La competencia socio-lingüística: componente que coloca a la lengua en el contexto social concreto en que se desarrolla el proceso de comunicación. Ser competente desde el punto de vista sociolingüístico implica tener la habilidad de utilizar e interpretar las formas lingüísticas apropiadamente con relación a una situación específica.

La competencia del discurso: habilidad de percibir y lograr coherencia de ideas separadas en patrones significativos de comunicación. Esta competencia está muy relacionada con la estructuración lógica del discurso, tanto en los códigos orales como los escritos.

La competencia estratégica: habilidad de utilizar estrategias verbales y no verbales para resolver problemas que pueden surgir en la comunicación. Se trata de saber qué hacer cuando no se es comprendido, saber expresar lo mismo de varias formas, y saber obtener de otras personas información que no se ha entendido total o parcialmente.

La competencia sociocultural: constituye el conocimiento y la habilidad de actuar con familiaridad en el contexto socio-cultural en el cual se utiliza la lengua. Se refiere a la habilidad de seleccionar los comportamientos apropiados desde el punto de vista cultural, no necesariamente lingüístico.

Finalmente, se asume lo planeado por Peña (2010) al expresar que las competencias expresan aquello que se espera que los estudiantes sean capaces de saber, saber hacer y saber ser, que modulan su carácter y formación en sentido amplio para convertirlos en profesionales y en ciudadanos autosuficientes. Es precisamente a lo que se aspira en el sistema educativo cubano.

\subsection{El aprendizaje cooperativo en la enseñanza aprendizaje del idioma inglés.}

El principal procedimiento para el aprendizaje es la comunicación y por lo tanto el lenguaje. Si se plantea una dinámica de aprendizaje cooperativo en la enseñanza aprendizaje del idioma inglés, se potencian las posibilidades comunicativas de esta lengua, haciendo uso de ellas en un contexto próximo a la realidad.

Willis (1996) hace una referencia clarificadora en este sentido al considerar que se crea una situación comunicativa real en el contexto escolar cuando hay una interacción entre los miembros de un grupo, con un objetivo común de resolución de una tarea. Es pues cuando la lengua es una herramienta 
más para el aprendizaje y el estudiante se ve con la necesidad de comunicarse para resolver la tarea en una situación en el aula próxima a la realidad. Por tal motivo el presente artículo consiste en utilizar el aprendizaje cooperativo para crear estos contextos reales que favorezcan el desarrollo de la competencia comunicativa en las clases de inglés.

Al ser heterogéneos los grupos para aplicar el aprendizaje cooperativo, favorece la cooperación, estableciendo relaciones de ayuda mutua que benefician a las dos partes implicadas. Díaz (2007) Además, trabajar con grupos en las clases de inglés permite atender a niños y niñas con un alto nivel de competencia lingüística (hijos de nativos, por ejemplo, que se aburren en las clases de inglés y suelen ser una pesadilla para los maestros) y niños con dificultades de aprendizaje generalizadas. Pérez (2004) citado por Cerda (2014).

Aunque en Cuba no es común encontrar hijos de nativos en las aulas, sí es normal encontrar estudiantes con un alto nivel de competencia, que al asignarle roles en un determinado grupo de aprendizaje cooperativo, pueden ser útiles durante la impartición de un determinado contenido pues el profesor se apoya en ellos para que intercambien sus conocimientos con el resto de los miembros del grupo.

El aprendizaje cooperativo aumenta las oportunidades de cada estudiante de poner en práctica su competencia comunicativa, y se basa en la suma de responsabilidades individuales, donde se debe asegurar la participación de todos. Así pues, se desarrollan habilidades interpersonales, en parte con la utilización de la lengua en situaciones muy próximas a la realidad cotidiana, como la práctica del diálogo y el consenso, que son claves en el desarrollo de la actividad.

Por lo que respecta al vocabulario, se activan los campos semánticos interiorizados por el estudiante. En cuanto a la gramática, se produce la gramaticalización de los fragmentos de lengua aprendidos como unidades completas de significado. Cuando los estudiantes se ven en una situación de explorar todos sus recursos, descomponen las unidades aprendidas y las vuelven a componer con un nuevo propósito. Cameron (2001). También tiene lugar la negociación de significado, que es positiva para las dos partes, la que debe encontrar los recursos para hacerse entender y la que va incorporando nuevos significados (Willis, 1996).

Según Cerdá (2014) citando a Brian (1990), el aprendizaje cooperativo se basa en las teorías de la psicología cognitiva del constructivismo y el aprendizaje significativo. Los estudiantes construyen el aprendizaje a partir del conocimiento previo y en la medida en que se produce un conflicto cognitivo, entre lo que se sabe y lo que se propone como nuevo. En este tipo de aprendizaje se habla también de conflicto socio cognitivo cuando este proceso se da en el seno de un grupo heterogéneo, con distintas experiencias previas y puntos de vista distintos. La resolución de este conflicto requiere de habilidades sociales y comunicativas, como lo requiere el desarrollo de la competencia comunicativa.

Se coincide con Johnson (1999) al plantear que el aprendizaje cooperativo le permite al docente alcanzar varias metas importantes al mismo tiempo. En primer lugar, lo ayuda a elevar el rendimiento de todos sus estudiantes, incluidos tanto los especialmente dotados como los que tienen dificultades para 
aprender. En segundo lugar, lo ayuda a establecer relaciones positivas entre los estudiantes, sentando así las bases de una comunidad de aprendizaje en la que se valore la diversidad. En tercer lugar, les proporciona a los estudiantes las experiencias que necesitan para lograr un saludable desarrollo social, psicológico y cognitivo. La posibilidad que brinda el aprendizaje cooperativo de abordar estos tres frentes al mismo tiempo, lo hacen superior a todos los demás métodos de enseñanza.

Además, con el aprendizaje cooperativo, el profesor pierde su rol protagónico y se convierte en un facilitador que organiza y facilita el aprendizaje en equipos, en lugar de limitarse a llenar de conocimientos las mentes de los estudiantes, sin saber si realmente entienden lo que les enseña. Para lograr este cambio, deberá emplear el aprendizaje cooperativo la mayor parte del tiempo.

El rol del docente, cuando emplea el aprendizaje cooperativo, es multifacético. Deberá tomar una serie de decisiones antes de abordar la enseñanza, explicar a los estudiantes la tarea de aprendizaje y los procedimientos de cooperación, supervisar el trabajo de los equipos, evaluar el nivel de aprendizaje de los estudiantes y alentarlos a determinar con qué eficacia están funcionando sus grupos de aprendizaje.

Si se compara esta labor del docente explicada por Johnson (1999) para emplear el aprendizaje cooperativo, con la de un profesor de inglés, es posible darse cuenta que en una clase de inglés se llevan a cabo estas mismas funciones, debido a que es necesario el trabajo en equipos, dúos, tríos o cuartetos para llevar a cabo las diferentes tareas de aprendizaje.

El aprendizaje cooperativo comprende tres tipos de grupos de aprendizaje:

1. Los grupos formales de aprendizaje cooperativo que funcionan durante un período que va de una hora a varias semanas de clase. En estos grupos, los estudiantes trabajan juntos para lograr objetivos comunes, asegurándose de que ellos mismos y sus compañeros de grupo completen la tarea de aprendizaje asignada. Cualquier tarea, de cualquier materia y dentro de cualquier programa de estudios, puede organizarse en forma cooperativa. Cualquier requisito del curso puede ser reformulado para adecuarlo al aprendizaje cooperativo formal.

Cuando se emplean grupos formales de aprendizaje cooperativo, el docente debe: (a) especificar los objetivos de la clase, (b) tomar una serie de decisiones previas a la enseñanza, (c) explicar la tarea y la interdependencia positiva a los estudiantes, (d) supervisar el aprendizaje de los estudiantes e intervenir en los grupos para brindar apoyo en la tarea o para mejorar el desempeño interpersonal y grupal de los estudiantes, y (e) evaluar el aprendizaje de los estudiantes y ayudarlos a determinar el nivel de eficacia con que funcionó su grupo.

2. Los grupos informales de aprendizaje cooperativo que operan durante unos pocos minutos hasta una hora de clase. El docente puede utilizarlos durante una actividad de enseñanza directa (una clase magistral, una demostración, una película o un vídeo, etc) para centrar la atención de los estudiantes en el material en cuestión, para promover un clima propicio al aprendizaje, para crear expectativas acerca del contenido de la clase, para asegurarse de que los estudiantes procesen cognitivamente el material que se les está enseñando y para dar cierre a una clase. 
La actividad de estos grupos informales suele consistir en una charla de tres a cinco minutos entre los estudiantes antes y después de una clase, o en diálogos de dos a tres minutos entre pares de estudiantes durante el transcurso de una clase magistral. Al igual que los grupos formales de aprendizaje cooperativo, los grupos informales le sirven al docente para asegurarse de que los estudiantes efectúen el trabajo intelectual de organizar, explicar, resumir e integrar el material a las estructuras conceptuales existentes durante las actividades de enseñanza directa.

3. Los grupos de base cooperativos que tienen un funcionamiento de largo plazo (por lo menos de casi un año) y son grupos de aprendizaje heterogéneos, con miembros permanentes, cuyo principal objetivo es posibilitar que sus integrantes se brinden unos a otros el apoyo, la ayuda, el aliento y el respaldo que cada uno de ellos necesita para tener un buen rendimiento escolar. Los grupos de base permiten que los estudiantes entablen relaciones responsables y duraderas que los motivarán a esforzarse en sus tareas, a progresar en el cumplimiento de sus obligaciones escolares (como asistir a clase, completar todas las tareas asignadas, aprender) y a tener un buen desarrollo cognitivo y social (Johnson, 1992).

Es necesario conocer que los verdaderos grupos de aprendizaje cooperativo son solo aquellos en los cuales a los estudiantes se les indica que trabajen juntos y ellos lo hacen de buen grado. Saben que su rendimiento depende del esfuerzo de todos los miembros del grupo. Cada miembro del grupo asume la responsabilidad, y hace responsable a los demás de realizar un buen trabajo. Todos los estudiantes tienen un mejor desempeño que si hubieran trabajado solos.

Si se trata de grupos en los cuales los estudiantes no tienen interés en hacerlo, creen que serán evaluados según la puntuación que se asigne a su desempeño individual, consideran que trabajarían mejor en forma individual y algunos tratan de sacar partido de los esfuerzos de sus compañeros más responsables, entonces no se puede considerar a este grupo como un grupo de trabajo cooperativo. Para emplear con eficacia el aprendizaje cooperativo, hay que distribuir cada tarea de tal manera que cada miembro de un grupo necesite del apoyo del otro para resolverla, si no se hace de esa manera, entonces no se ha creado un grupo de aprendizaje cooperativo.

Para organizar las clases de inglés de modo que los estudiantes realmente trabajen en forma cooperativa para el logro de la competencia comunicativa, el profesor debe tener en cuenta los elementos básicos que hacen posible la cooperación planteados por Johnson (1992).

1. Tomar sus clases, programas y cursos actuales, y organizarlos cooperativamente.

2. Diseñar clases cooperativas que se ajusten a sus propias necesidades y circunstancias pedagógicas, a sus propios programas de estudios, materias y estudiantes.

3. Diagnosticar los problemas que puedan tener algunos estudiantes para trabajar juntos, e intervenir para aumentar la eficacia de los grupos de aprendizaje. 
Según Johnson (1992), para que la cooperación funcione bien, hay cinco elementos esenciales que deberán ser explícitamente incorporados en cada clase.

El primer y principal elemento del aprendizaje cooperativo es la interdependencia positiva. El docente debe proponer una tarea clara y un objetivo grupal para que los estudiantes sepan que habrán de hundirse o salir a flote juntos. Los miembros de un grupo deben tener en claro que los esfuerzos de cada integrante benefician a todos los miembros de igual manera. Esta interdependencia positiva crea un compromiso con el éxito de otras personas, además del propio, lo cual es la base del aprendizaje cooperativo. Sin interdependencia positiva, no hay cooperación.

El segundo elemento esencial del aprendizaje cooperativo es la responsabilidad individual y grupal. El grupo debe asumir la responsabilidad de alcanzar sus objetivos, y cada miembro será responsable de cumplir con la parte del trabajo que le corresponda. Nadie puede aprovecharse del trabajo de otros. La responsabilidad individual existe cuando se evalúa el desempeño de cada estudiante y los resultados de la evaluación son transmitidos al grupo y al individuo a efectos de determinar quién necesita más ayuda, respaldo y aliento para efectuar la tarea en cuestión.

El tercer elemento esencial del aprendizaje cooperativo es la interacción estimuladora, preferentemente cara a cara. Los estudiantes deben realizar juntos una labor en la que cada uno promueva el éxito de los demás, compartiendo los recursos existentes y ayudándose, respaldándose, alentándose y felicitándose unos a otros por su empeño en aprender.

El cuarto componente del aprendizaje cooperativo consiste en enseñar a los estudiantes algunas prácticas interpersonales y grupales. El aprendizaje cooperativo es complejo porque requiere que los estudiantes aprendan tanto las materias escolares (ejecución de tareas) como las prácticas interpersonales y grupales necesarias para funcionar como parte de un grupo (trabajo de equipo). Los miembros del grupo deben saber cómo ejercer la dirección, tomar decisiones, crear un clima de confianza, comunicarse y manejar los conflictos, y deben sentirse motivados a hacerlo.

El quinto elemento fundamental del aprendizaje cooperativo es la evaluación grupal. Esta evaluación tiene lugar cuando los miembros del grupo analizan en qué medida están alcanzando sus metas y, manteniendo relaciones de trabajo eficaces. Los grupos deben determinar qué acciones de sus miembros son positivas o negativas, y tomar decisiones acerca de cuáles conductas conservar o modificar.

El empleo del aprendizaje cooperativo requiere una acción disciplinada por parte del docente. Los cinco elementos básicos no sólo son características propias de los buenos grupos de aprendizaje, también representan una disciplina que debe aplicarse rigurosamente para producir las condiciones que conduzcan a una acción cooperativa eficaz.

Una vez creados los diferentes grupos de aprendizaje cooperativo, son múltiples las técnicas de aprendizaje cooperativo que al utilizarse adecuadamente permiten el desarrollo de la competencia comunicativa en inglés. Por ejemplo: 
El rompecabezas: es el modo de hacer que los estudiantes sean interdependientes. Es ofrecer la información distribuida en distintas partes, como si fueran las piezas de un rompecabezas. Con este método, cada estudiante obtiene una parte de la información necesaria para realizar la tarea. Los miembros del grupo son responsables de conocer a fondo la información que les corresponde, transmitírsela a los demás y aprender la información presentada por los otros miembros del grupo. Johnson (1992)

En varias observaciones realizadas a diferentes clases de inglés se corroboró que existen estudiantes que tienen desarrollada una habilidad más que otra, es decir, hay estudiantes que escriben muy bien y dominan la gramática, pero oralmente presentan dificultades y viceversa.

\section{Metodología:}

La técnica en este caso consiste en asignar una situación comunicativa para desarrollar un diálogo aplicando diferentes funciones comunicativas, el léxico y la gramática adecuada. Durante el desarrollo de la actividad cada integrante del grupo será entonces responsable de entrenar a los demás miembros en la habilidad que mejor dominan de acuerdo al contexto de que se trate. Como cada miembro necesita el entrenamiento de sus compañeros para exponer el diálogo tanto de forma oral como escrita, habrá una interdependencia entre todos los integrantes del grupo y todos participarán en la actividad al tratarse de un diálogo aplicable a una situación de su realidad objetiva, en el cual cada uno desempeña un determinado rol.

Otra variante del rompecabezas puede ser: asignar la escritura de un texto. En este caso se le propone al equipo un tema con diferentes ideas que lo sustentan. Luego se le da la tarea a cada miembro para que desarrolle las diferentes ideas y al final todos juntos deben elaborar el texto con las diferentes ideas desarrolladas y exponer el texto de forma oral y escrita.

Asignar roles: es una de las maneras más eficaces de asegurarse de que los miembros de un grupo cooperativo trabajen juntos sin tropiezos y en forma productiva. Esto se aplica constantemente durante la exposición de diálogos y también se puede aplicar al trabajar la comprensión de un texto.

\section{Metodología:}

Orientar la lectura de un texto y contestar preguntas sobre el mismo, por ejemplo:

1. Un miembro del grupo lee el texto en voz alta mientras los demás siguen la lectura con la vista. A través de la coevaluación se corrigen los posibles errores cometidos en la lectura. Es importante declarar al inicio de la actividad los indicadores que permiten la realización de una lectura correcta. Estos pueden ser: uso correcto de la pronunciación, adecuada entonación de las oraciones interrogativas y exclamativas, realización de las pausas necesarias, empleo correcto de las uniones de palabras, entre otras que el profesor considere necesarias.

2. Otro miembro del grupo lee la primera pregunta. 
a. Cada estudiante ofrece posibles respuestas.

b. Se designa a un estudiante de alto rendimiento para llevar el registro de respuestas aceptables.

c. El grupo decide cuál es la mejor de las respuestas teniendo en cuenta la adecuada pronunciación y uso correcto de la gramática y el vocabulario.

d. El estudiante encargado de llevar el registro de respuestas aceptables pide a uno o más miembros del grupo que expliquen por qué la respuesta elegida es la mejor.

3. Se repite el segundo paso para cada pregunta.

4. Después de haber contestado todas las preguntas, el grupo realiza un resumen del texto y designa a uno para su exposición oral.

El debate: éste puede aplicarse para ofrecer opiniones sobre un tema determinado, función comunicativa importante en una clase de inglés.

\section{Metodología:}

Para ofrecer opinión sobre un tema determinado en una clase de inglés resulta necesario redactar un párrafo sobre los aspectos positivos, otros sobre los aspectos negativos y finalmente redactar otro párrafo para ofrecer las conclusiones. Para ello se sugieren las siguientes acciones:

1. Elegir un tema cuyo contenido puedan manejar los estudiantes y sobre el cual puedan elaborarse al menos dos posiciones (aspectos positivos y aspectos negativos).

2. Preparar los materiales didácticos de modo que los miembros del grupo sepan qué posición les ha sido asignada y dónde pueden encontrar información para fundamentarla.

3. Formar grupos de cuatro miembros y dividirlos en dos pares, uno para expresar los aspectos positivos y otro para expresar los aspectos negativos. Hay que poner de relieve el objetivo cooperativo de llegar a un consenso sobre el tema y redactar un texto grupal sobre el que todos los miembros serán evaluados.

4. Asignar a cada par la tarea cooperativa de aprender su posición, así como los argumentos e información que la fundamenten.

5. Hacer que cada par presente sus aspectos al otro. El grupo discutirá el tema, evaluando críticamente los aspectos positivos y negativos y sus fundamentos para luego todos juntos agregar un último párrafo con la opinión global sobre el tema.

7. Finalmente, hacer que los miembros del grupo expongan el tema tanto de forma oral como escrita.

El cierre a una clase: Es conveniente que los estudiantes se reúnan en grupos reducidos y discutan lo que aprendieron, pues esto les permite formular lo que saben y explicárselo a otros. 


\section{Metodología:}

1. Hacer que los estudiantes se reúnan en sus grupos cooperativos (o formar grupos nuevos de dos o tres miembros).

2. Asignar a los estudiantes la tarea de resumir los contenidos de la clase y lo que ellos aprendieron. La meta cooperativa es elaborar un solo trabajo en el que se describan el contenido de la clase, las cinco cuestiones principales que se aprendieron y dos preguntas que los miembros del grupo quieran hacer sobre la clase.

3. Finalmente se escoge a un miembro del grupo para exponer el resumen.

Resulta importante conocer que el aprendizaje cooperativo requiere una evaluación, la cual hoy en día es una etapa comunicativa, un momento en que se enfatiza la evaluación del uso del lenguaje en lugar de la forma. La gramática, la pronunciación y el vocabulario deben ser evaluados, pero en el contexto y asociados al significado y la función. Además, estos aspectos del lenguaje no deben pesar más que las habilidades comunicativas.

El profesor no debe olvidar que el sistema del lenguaje es solo un medio para cumplir un objetivo: la comunicación. Por lo tanto, los profesores deben evaluar, principalmente, lo que los estudiantes pueden hacer con el idioma y cómo lo utilizan en contexto. Los profesores no se deben conformar con ser solo ellos lo que evalúan. Es necesario establecer indicadores con los estudiantes desde el primer día de clase para que también puedan autoevaluarse y coevaluarse. De esta manera se desarrolla una actitud crítica ante el aprendizaje. Se coincide con Peña (2010) al plantear que la medida del éxito de cada actividad, depende en gran parte de establecer indicadores y determinar, mediante los mismos y previa comunicación a los estudiantes, la capacidad de cada estudiante o de cada grupo.

Torrego y Negro (2012) proponen otros métodos de aprendizaje cooperativo, los cuales también se pueden aplicar a las clases de inglés. Entre ellos se encuentran:

Tutoría entre iguales (peer tutoring): con el fin que el estudiante tutor aprenda enseñando y el tutorado aprenda también al recibir una ayuda personalizada de su compañero.

Enseñanza recíproca: se trata de mentes (roles) que trabajan en equipo, desarrollando funciones que una mente experta realiza individualmente.

Grupo de investigación: la clase convertida en una comunidad científica, trabaja un tema dividiéndolo en diferentes subtemas de los que se encarga cada equipo.

\section{RESULTADOS}

La investigación se orientó hacia la muestra antes referida y comenzó con la aplicación de diferentes métodos de investigación. 
En la encuesta aplicada a los estudiantes de la muestra se corroboró que el 66,7\% entiende lo expresado por el profesor y sus compañeros, pero no pueden interactuar oralmente de manera espontánea porque no encuentran la gramática y el léxico adecuado para hacerlo. El 38,3\% consideró que se preocupan por los errores que pudieran cometer, por lo sienten temor a la hora de participar en las clases. El 90,0\% desea realizar actividades organizadas en grupos que permitan la interacción de unos con otros y se ayuden mutuamente durante el desarrollo de las clases.

El $100 \%$ de los profesores entrevistados tienen suficiente experiencia en el nivel universitario, conocen los diferentes componentes de la competencia comunicativa a desarrollar en los estudiantes de cada año académico, crean diferentes grupos para desarrollar las actividades en las clases, pero no están conscientes de que en muchas ocasiones están trabajando haciendo uso de técnicas de aprendizaje cooperativo. De ahí la significación práctica del presente artículo.

Para evaluar la efectividad de las técnicas de aprendizaje cooperativo elaboradas se aplicó un preexperimento. El mismo comenzó con la aplicación de una prueba pedagógica inicial. La misma arrojó que los estudiantes exhiben un deficiente desempeño cognitivo, ilustrado a través de las considerables limitaciones de la competencia estratégica, discursiva y gramatical, es decir, utilizaron inadecuadamente los patrones gramaticales estudiados y no encontraron el léxico adecuado a emplear para entender a los demás o hacerse entender en un contexto determinado.

Luego del estudio de los referentes teóricos relacionados con el tema en cuestión, se elaboraron diferentes técnicas de aprendizaje cooperativo y se aplicaron en las clases durante dos unidades del programa de segundo año de la carrera Lenguas Extranjeras, Inglés. Se pudo corroborar que los estudiantes de la muestra seleccionada sufrieron cambios significativos en relación con el desarrollo de la competencia comunicativa. Esto se corroboró con la aplicación de una prueba de salida. En este caso los resultados fueron superiores si se comparan con los de la prueba pedagógica aplicada inicialmente.

Cada prueba consistió en entrevistar a los estudiantes a través de la cual debían interactuar con el profesor y sus compañeros ofreciendo información personal, narrando historias cortas, describiendo lugares y personas, ofreciendo opiniones sobre un tema determinado, entre otras tipologías de ejercicios. Se evaluó a cada estudiante con 2, 3, 4 y 5 puntos, teniendo en cuenta cada una de los componentes de la competencia comunicativa ofrecidos en este artículo. Finalmente se consideraron los siguientes indicadores:

Rendimiento académico alto: estudiantes con 4 y 5 puntos.

Promoción adecuada: más del $75 \%$ de estudiantes aprobados.

Calidad en el aprendizaje alta: estudiantes con 4 y 5 puntos.

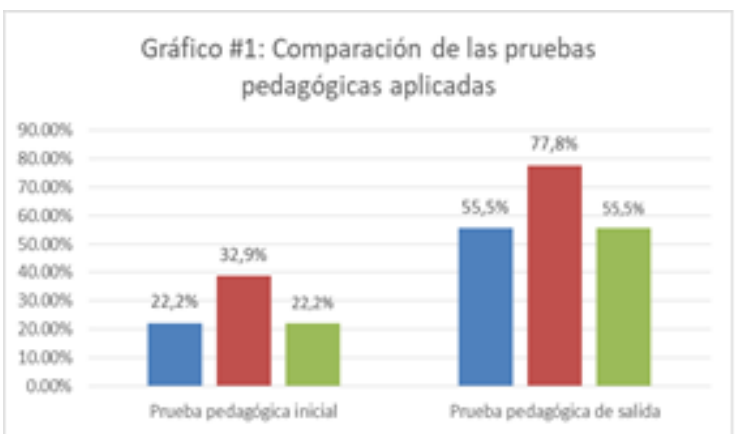

Figure 1 Comparación de las pruebas pedagógicas aplicadas 
La mayoría de las técnicas aplicadas alcanzaron un alto nivel de aceptación tonto por los estudiantes como por los profesores, pues durante su aplicación hubo una participación activa y consciente en las clases, todos los estudiantes intercambiaron de forma oral entre sí y con los profesores mediando entre ellos la comunicación como actividad humana, lo cual le permitió utilizar el idioma en diferentes contextos, sobre todo, en aquellos que los acercó a la realidad que pudieran enfrentar en sus futuras profesiones. Además, garantizó dinamismo en las clases y favoreció la fijación del nuevo material lingüístico introducido, incluyendo una amplia gama de vocabulario, así como el desarrollo de los componentes competencia gramatical, estratégica y discursiva y sociocultural de forma activa, consciente y práctica.

La enseñanza tradicional pensaba que las interacciones entre estudiantes no tenían interés educativo. Ahora, sin embargo, sabemos que si estas se establecen apropiadamente pueden dar lugar a un entorno de aprendizaje muy valioso. Por tanto, conviene subrayar que el aprendizaje cooperativo no tiene un uso temporal, sino que responde a una necesidad pedagógica y social (Torrego y Negro, 2012).

\section{CONCLUSIONES}

El objetivo de la enseñanza aprendizaje del idioma inglés como lengua extranjera en Cuba es desarrollar la competencia comunicativa. El enfoque que así lo permite es el comunicativo al reconocer como su objetivo el logro de dicha competencia. El aprendizaje cooperativo aumenta las oportunidades de cada estudiante de poner en práctica su competencia comunicativa, pues de la manera en que se organiza y se pone en práctica permite la interacción de los estudiantes en diferentes contextos comunicativos. El empleo del aprendizaje cooperativo por parte de un profesor de inglés es relativamente fácil de poner en práctica en sus clases, ya que en una clase de inglés se trabaja en equipos, parejas, se asignan roles, se emplea la autoevaluación y la coevaluación, aspectos que también están presentes cuando se aplica el aprendizaje cooperativo. Existen diversos tipos de grupos de aprendizaje cooperativo que permiten tener un buen desarrollo cognitivo y social. Las técnicas de aprendizaje cooperativo sugeridas son efectivas para el desarrollo de la competencia comunicativa en las clases de inglés; así lo demostró el pre-experimento realizado.

\section{REFERENCES}

Brown, D. (1994). Teaching by Principles. Prentice Hall Regents, New Jersey.

Brian, I. (1990). El aprendizaje cooperativo. Sharan, Pamplona: Departamento de Educación y Cultura.

Cameron, L. (2001). Teaching Languages to Young Learners. Cambridge: Cambridge University Press.

Cerdá Vallés, C. (2014). El aprendizaje cooperativo para el desarrollo de la competencia comunicativa oral en lengua inglesa de primaria. Consultado 2 de abril de 2019. http://encuentrojournal.org/textcit.php?textdisplay=466

Chomsky, N. (1965). Aspects of the Theory of Syntax. Cambridge: MIT Press. 
DeVries, D. (1974). Student Teams and Learning Games: Their Effects on Cross-Race and Cross-Sex Interaction, Journal of Educational Psychology. Díaz Aguado, M. J. (2007). Educación intercultural y aprendizaje cooperativo. Madrid: Ediciones Pirámide.

Font Milán, S. (2005). Una propuesta metodológica basada en el enfoque comunicativo y la enseñanza problémica para la asignatura inglés en la secundaria básica con apoyo del video. Tesis en opción al grado de Doctor en Ciencias Pedagógicas. UCP: Enrique José Varona. La Habana.

Hymes, D. (1972). On Communicative competence. En J.B. Pride and J. Homes, Sociolinguistics. Harmonds worth, Penguin.

Johnson, D. W. (1991). Active Learning: Cooperation in the College Classroom, Edina, Minnesota, Interaction Book Company.

Johnson, D. W. (1992). Advanced Cooperative Learning, Edina, Minnesota, Interaction Book Company.

Johnson, D. W. (1999). El aprendizaje cooperativo en el aula. Buenos Aires: Paidós.

Nunan, D. (1989). Designing Tasks for the Communicative Classroom. Cambridge University Press, U.K.

Peña, J.D. (2010). El aprendizaje cooperativo y las competencias. Consultado 9 de enero de 2020. El aprendizaje cooperativo y las competencias https://dialnet.unirioja.es/servlet/articulo

Pérez, P. y Roig, V. (2004). Enseñar y aprender inglés en educación infantil y primaria. Barcelona: ICEHORSORI.

Richards, Jack, C. (2001). Curriculum Development in Language Teaching. Cambridge Language Education. Cambridge University Press, United Kingdom.

Richards, Jack, C. (2001). Approaches and Methods in Language Teaching, Cambridge University Press, U.K.

Savignon, S. (1983). Communicative Competence. Theory and Classroom Practice. Texts and contexts in second language learning. Reading, Mass. Addison-Wesley.

Sharan, S. (2004). El desarrollo del aprendizaje cooperativo a través de la investigación en grupo. Sevilla: Publicaciones M.C.E.P. SEVILLA. Torrego, J.C. y Negro, A. (coords.) (2012). Aprendizaje cooperativo en las aulas. Fundamentos y recursos para su implantación. Madrid: Alianza Ed.

Vygotsky, L.S. (1984). Obras completas. Tomo 4, Ed. Pedagogía, Moscú.

Willis, J. (1996). A Framework for Task-Based Learning. Essex: Longman. 